\title{
Middleware for the use of storage in communication ${ }^{2}$
}

\author{
Micah Beck ${ }^{\mathrm{a}}$, Dorian Arnold ${ }^{\mathrm{b}}$, Alessandro Bassi ${ }^{\mathrm{c}}$, \\ Fran Berman $^{\text {d }}$, Henri Casanova ${ }^{\text {d }}$, Jack Dongarra ${ }^{\text {a,* }}$, \\ Terry Moore ${ }^{\text {a }}$, Graziano Obertelli e , James Plank ${ }^{\text {a }}$, \\ Martin Swany ${ }^{\mathrm{e}}$, Sathish Vadhiyar ${ }^{\text {a }}$, Rich Wolski ${ }^{\text {c }}$ \\ a Department of Computer Science, The University of Tennessee, 1122 Volunteer Boulevard, \\ Suite 203, Knoxville, TN 37996-3450, USA \\ ${ }^{\mathrm{b}}$ Computer Sciences Department, University of Wisconsin, 1210 West Dayton Street, \\ Madison, WI 53706, USA \\ ${ }^{c}$ RESO-LIP, ENS Lyon, 46, Allée d'Italie, 69364 Lyon Cedex 07, France \\ ${ }^{\mathrm{d}}$ Department of CDS/CSE, University of California, San Diego, \\ 9500 Gilman Drive, La Jolla, CA 92093-0114, USA \\ e Department of Computer Science, University of California, \\ Santa Barbara, Santa Barbara, CA 93106, USA
}

Received 11 October 2001; received in revised form 29 April 2002; accepted 17 June 2002

\begin{abstract}
The Logistical Computing and Internetworking (LoCI) project is a reflection of the way that the next generation internetworking fundamentally changes our definition of high performance wide area computing. A key to achieving this aim is the development of middleware that can provide reliable, flexible, scalable, and cost-effective delivery of data with quality of service guarantees to support high performance applications of all types. The LoCI effort attacks this problem with a simple but innovative strategy. At the base of the LoCI project is a richer view of the use of storage in communication and information sharing.
\end{abstract}

(c) 2002 Elsevier Science B.V. All rights reserved.

Keywords: Internetworking; Wide area computing; Middleware; Information sharing; Grid computing; Logistical computing

\footnotetext{
Work supported in part by the NSF/NGS GRANT \#NSF EIA-9975015, and NSF GRANT ACI9876895.

${ }^{*}$ Corresponding author. Fax: +1-8659748296.

E-mail address: dongarra@cs.utk.edu (J. Dongarra).
} 


\section{Introduction}

At the base of the Logistical Computing and Internetworking (LoCI) research is a richer view of the use of storage in communication. Current approaches to quality of service (QoS) rely on the standard end-to-end model of communication: the state of network flow is maintained at the end nodes and not in the network. By contrast, our concept of logistical $Q o S$ is a generalization of the typical model that permits state management within the networking fabric itself, via a much more flexible control of message buffering, in order to achieve QoS delivery without difficult end-to-end requirements. For example, whenever data is available to be sent well before it needs to be received, it can be staged, i.e., moved in advance and stored in a location "close" to the receiver for later delivery. We define such strategies that employ storage in communication, as logistical network computing, and the main purpose of the LoCI project is to investigate and test the central conjecture of logistical network computing:

\section{If}

(1) distributed network storage is made available as a resource and is flexibly schedulable and

(2) communication, computational, and storage resources can be predictably allocated for coscheduling,

Then advanced applications can be implemented on computational grids with higher performance and/or lower overall use of communication, computational, and storage resources.

The structure of our research in the LoCI program reflects the parts of this conjecture, which in turn represent the fundamental elements of logistical network computing. To create a research-computing environment that enables us to allocate communication, computation, and storage resources for coscheduling, we combine four technologies from the world of computational grids:

- Internet Backplane Protocol (IBP) [1] is primitive middleware that supports a layer of network storage, implemented as a system of buffers exposed for direct scheduling, that advanced applications can use to leverage state management for high-performance.

- Network Weather Service (NWS) [2] enables us to predict the ability of the network to respond to data movement requests over time.

- NetSolve [3] provides a programming environment that facilitates the analysis of program dependences, expressed in the form of dependence flow graphs, to understand an application's inherent communication requirements. A major component of LoCI research is to identify and provide opportunities for extracting scheduling information from applications.

- Application level scheduling (AppLeS) [4] is enabling us to derive an efficient schedule that meets those communication requirements. Once the scheduling 
information is made available, mapping the computation, network and storage resources of the application to the Grid resources, subject to current and predicted resource conditions, is a difficult problem. AppLeS is the leading instance of a range of approaches we are exploring under LoCI.

These Grid technologies have focused, primarily, on the control of compute and network resources to achieve high-performance distributed execution. Logistical computing adds the control of storage to form a comprehensive Grid infrastructure. By exposing more of the underlying storage structure of the network and maximizing its exploitation in scientific applications, our research is moving network computing towards the physical and logical limits of the underlying technology, as is found in more mature areas of computer engineering.

\section{Logistical network computing and explicit storage control}

Our architectural analysis of high performance network computing derives from an analogy with the architecture of modern pipelined microprocessors. The fundamental elements of modern processor architecture are:

- Buses and functional units which move and transform data, and

- Memory and cache, registers and pipeline buffers that store data.

With these mechanisms in place, the programming interface can then schedule the execution of a program in a way that achieves maximum performance. Careful control of data at the various stages of an execution pipeline is necessary to ensure high performance levels. It is our belief that Grid programs (or the Grid middleware) must analogously control program state as it traverses the Grid.

Another important difference between modern RISC and VLIW architectures and the CISC architectures of the 70s and $80 \mathrm{~s}$ is that instructions are predictable because they model the processor pipeline very directly. All elements of the pipeline behave in a completely deterministic fashion except for the cache, which is statistically predictable.

In our model of logistical network computing, the fundamental elements are

- Predictable networking and computation which move and transform data, and

- Storage that is accessible from the network.

Using these elements, the programming interface can then schedule the execution of a program in a way that achieves maximum performance. One important difference between logistical network computing and traditional methods is that it is based on global scheduling expressed at the programming interface but implemented by local allocation throughout the network. Traditional approaches express at the programming interface only complex higher-level operations defined in terms of the endpoints, encapsulating the complexity of the network. The result is that it is much harder to implement predictable operations. 


\section{The Internet Backplane as middleware for next generation software}

In order to experiment with logistical network computing, some mechanism for the management of storage is required. Staging can be implemented at many possible levels in the application or operating system, and as with all software architecture decisions, the tradeoffs are complex, involving many factors including compatibility of interfaces, administrative convenience and performance.

Most network computing environments are fairly self-contained in the sense that data flows only between processors which host compute servers, and so it is possible to implement data depots and storage management as part of the compute server. Under this approach staging is accessible only to a single network computing domain, so that the management of storage is not shared between environments (e.g. NetSolve [5], Globus [6], and Legion [7]) or between instances of a single environment. Such sharing is important because it allows storage to be managed as an aggregate resource rather than as several smaller pools, and because it allows performance-enhancing services such as caching to be implemented in an application- and environment-neutral manner.

The middleware approach is to abstract a model of state management from the particular computing environment and to define it to be a lower level service. It is possible to implement that service in a user-level library, in a daemon process or in kernel network drivers that reach lower into the protocol stack. In fact, the functionality may ultimately be spread across these architectural levels, and could ultimately be supported by modifications to the network infrastructure itself.

A key innovation of the LoCI project is the implementation of a software mechanism for distributed data staging, called the IBP, a middleware service implemented by TCP/IP connections to daemon processes, in the style of FTP and NFS.

\section{An overview of the Internet Backplane Protocol}

Fundamentally, IBP is designed to allow much freer control of buffer management at distributed storage depots through a general, but non-traditional scheme for naming, staging, delivering and protecting data. To address the needs of new Grid applications IBP diverges from the standard storage management systems (e.g., distributed file systems or databases) in three fundamental ways, which we consider in turn.

4.1. IBP serves up both writable and readable storage to anonymous clients as a widearea network resource

The Internet is a mainly stateless communication substrate that serves up two kinds of network resources to its generic and unauthenticated clients: read-only storage through anonymous FTP and the Web, and remote processing servers that connect to clients via two-way ASCII communication pipes with Telnet. There are 
projects that are trying to enlarge this resource space, such as Jini, NetSolve, and active disk and network movement. IBP enlarges this resource space by focusing on storage, namely writable storage. The benefits of offering writable storage as a network resource are numerous:

- QoS guarantees for networking can be met more easily when the intermediate routing nodes can store the communication buffers.

- Resource schedulers can include the staging of data near the processing resources for better resource utilization and better scheduling.

- Content services can be enhanced with both client and server-driven replication strategies (including, but not limited to caching, content push, multicast support, and replica management) for improved performance.

- A ubiquitous foundation for achieving fault-tolerance may be achieved.

Currently, most strategies for achieving the above benefits are ad hoc workarounds of the existing Internet architecture.

\subsection{IBP allows for the remote control of storage activities}

Storage managed by IBP may be viewed as files or buffers, located on reliable storage, in RAM, or perhaps on an active disk. IBP allows a user or processing entity to both access and manage these storage entities remotely, without being involved in the actual manipulation of the bytes. We present three general categories of how this improves application performance and flexibility below.

As an illustration in Fig. 1, consider the generation of sensor data in NWS. NWS generates a tremendous amount of performance data in order to make its predictions. It is not clear when the data is being collected whether or not it will be used (i.e., clients might not request predictions for a few minutes). Therefore it is optimal to store the data in a location close to the sender so that the storing is optimized. Sending the data to clients is less optimal, but that is a more infrequent operation. Ideally, of course, the data is stored on the machine being monitored, but that may not be possible. Storing it nearby in IBP is the next best alternative.

A similar example is checkpointing computations within NetSolve for fault-tolerance [8]. Since checkpoints may never be used, NetSolve would like to optimize the act of checkpointing. Obviously, it is not a good idea to store the checkpoint on the

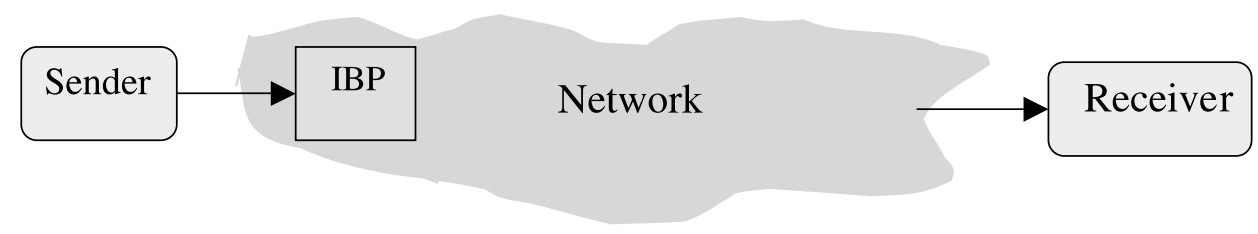

Fig. 1. IBP: keeping data close to sender. 


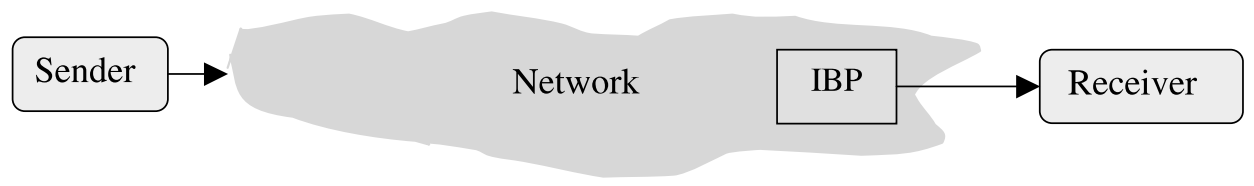

Fig. 2. IBP: place data close to receiver.

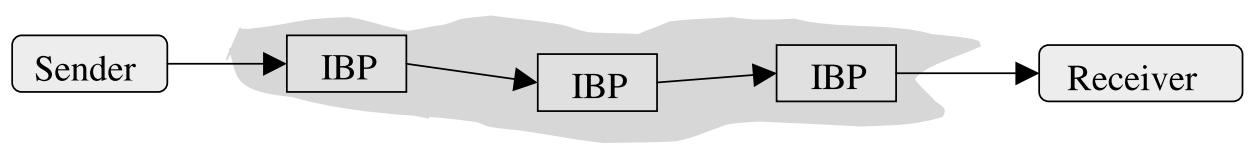

Fig. 3. IBP: utilizing storage throughout.

compute server, because if the server fails, the checkpoint may not be available (since the server is down). IBP thus allows the servers to checkpoint "nearby", which allows for an optimal balance of performance and fault-tolerance.

In Fig. 2, the data is put close to the receiver so that the overhead of receiving is low. Standard performance optimizations such as staging and caching fall into this category, and are well-known enough to require no further elaboration.

In Fig. 3, storage is used in the network to explicitly route a message. This obviously improves the performance of broadcast messages. Additionally, it helps with intermediate link failures. With standard end-to-end networking, one has to resend packets from the sender if any link fails. With intermediate storage, the resend only has to happen on the failed link. Finally, with intermediate storage, a user can do explicit routing, which may be much more effective than standard Internet routing [9].

\subsection{IBP decouples the notion of user identification from storage}

Typically, storage systems require authentication for any access that uses a persistent resource, whereas networking has no persistent resources and so can rely on security implemented at the end-points. IBP treats all storage as if it were a communication buffer by offering up writable storage on the network to unauthenticated clients. That clients are unauthenticated does not mean that the system is chaotic or without safeguards. IBP allows the owner of a storage server to define how much storage to serve for IBP and how that storage should be served. In particular, IBP file allocation includes the notion that files may have a limited lifetime before they are removed by the IBP system. Each file is accessed through a unique storage capability so that access can be restricted without authentication. In addition, an IBP file may be allocated as volatile, meaning that the IBP server may revoke the storage at any time. Such a system strikes a balance between offering the benefits of writable storage on the network, and making sure that the owner of such storage has the ability to reclaim it when desired. 


\section{Logistical mechanisms}

The two key logistical mechanisms that we are designing are the IBP, which allows us to express logistical data movement, and the NWS that allows us to predict the effects of future requests for data movement.

\subsection{The Internet Backplane Protocol API}

We have defined and implemented a client API for IBP consisting of seven procedure calls, and a server daemon software that makes local storage available for remote management. Currently, connections between clients and servers are made through TCP/IP sockets.

IBP client calls may be made by any process that can connect to an IBP server. IBP servers do not require administrative privileges to install and operate, so IBP has the flavor of software such as PVM [10] that can leverage the privileges of ordinary users to create a distributed computing platform. IBP servers can implement various storage allocation policies in order to control the local impact. For example, the IBP server may be allowed to allocate spare physical memory, or it may be directed to only allow the allocation of unused disk space and to revoke that allocation in favor of local use when necessary. Alternatively, the IBP server may enforce only time-limited allocations, where the storage is automatically revoked after a set time period. These features manage the local impact of allowing allocation of local resources through IBP.

Each IBP server allocates storage in the form of append-only byte arrays. There are no directory structures or file names (this structure can be layered on top of IBP through the use of a directory server such as Globus' MDS). Clients initially allocate storage through a request to an IBP server. If the allocation is successful, the server returns three capabilities to the client, one for reading, one for writing, and one for management. These capabilities can be viewed as names that are assigned by the server and are meaningful only to IBP. The contents of the capability can be obscured cryptographically in order to implement a basic level of security. In order to achieve high performance, applications can pass and copy capabilities among themselves without coordinating through IBP.

IBP's API and several logistical network computing applications are described in detail in other documents [11,12].

\subsection{The Network Weather Service: monitoring resources for logistical scheduling}

While IBP provides the mechanisms that allow applications to exploit logistical network computing, resource usage must be carefully scheduled or application performance will suffer. To make these decisions the scheduler must predict the future performance of a set of resources. We use the NWS [13] to make these predictions based on the observed performance history of each resource.

The NWS periodically monitors available resource performance by passively and actively querying each resource, forecasts future performance levels by statistically 
analyzing monitored performance data in near-real time, and reports both up-to-date performance monitor data and performance forecasts via a set of well-defined interfaces.

The monitor interface is easily extensible; currently implemented monitors include TCP/IP latency and bandwidth, CPU load, and Globus GRAM process start times [14]. Monitor traces are presented as time series to a set of NWS forecasting models that make short-term performance predictions levels. Both forecast values and accuracy measures are reported for each resource and performance characteristic. Using this accuracy information, schedulers can gauge the value of individual forecasts and use this valuation to exploit different risk strategies. Forecast and forecast-quality data is published via C-language interfaces for access by dynamic schedulers.

It is the function of logistical scheduling to compose intermediate network and storage resources into an end-to-end "path" that supports a specified QoS. Our schedulers will rely on NWS performance forecasts to identify, dynamically, resource compositions that meet the QoS specifications of different, and potentially competing, Grid applications. A key research question that we are addressing concerns the degree to which NWS predications may be effectively composed to produce an overall predication.

\section{NetSolve as an environment for experimentation in logistical scheduling}

NetSolve is a client, server and agent system in which the client uses simple procedure calls to solve some problems remotely through the services hosted by a NetSolve server. The agent acts as a coordinator of the NetSolve Grid maintaining the state information of the different components and acting as a global scheduler.

The concept of storage provided by LoCI through IBP depots can be used in a variety of ways in the NetSolve framework.

1. NetSolve involves communication of data between the client and the servers. This data can be cached in an IBP depot located near the server(s) in situations where the NetSolve client calls a number of NetSolve problems with the same data.

2. In cases where the NetSolve user is not interested in the intermediate results, these intermediate results can be stored in the IBP depots for later use by the NetSolve servers during next phase of the computation.

3. In a collaborative project, where the NetSolve users interact to solve a large application, the LoCI framework provided through the IBP depots can be used to share the NetSolve results among the various users.

Mechanisms have been implemented in NetSolve where the user can invoke NetSolve calls to allocate storage in the IBP depots, encapsulate the storage in the form of an object and pass these objects in the NetSolve calls to solve problems. Thus the input data to a NetSolve problem can be stored in an IBP depot before the user makes the NetSolve call to solve the problem and the output data from the NetSolve servers can be directed to an IBP depot. The NetSolve calls that deal with the LoCI 
storage infrastructure are in turn implemented in terms of the simple API calls provided by LoCI. Though the NetSolve users can achieve the desired effect by directly invoking the LoCI API, NetSolve provides the abstraction in order to experiment with different storage infrastructures.

In the following experiment, we illustrate the use of IBP in NetSolve to cache input and intermediate results. The problem involves multiplication of two complex matrices, $A$ and $B$ to form a complex matrix $C$, followed by solving a complex system of equations involving $C$ and a known matrix $Y$, i.e.,

1. $C=A * B$

Implemented as

$$
\begin{aligned}
& C_{\mathrm{r}}=A_{\mathrm{r}} B_{\mathrm{r}}-A_{\mathrm{i}} B_{\mathrm{i}} \\
& C_{\mathrm{i}}=A_{\mathrm{i}} B_{\mathrm{r}}+A_{\mathrm{r}} B_{\mathrm{i}}
\end{aligned}
$$

where $A_{\mathrm{r}}, B_{\mathrm{r}}$ and $C_{\mathrm{r}}$ are the real parts of the matrices and $A_{\mathrm{i}}, B_{\mathrm{i}}$ and $C_{\mathrm{i}}$ are the imaginary parts of the matrices.

2. $C * X=Y$

where $Y$ is a known complex matrix and $X$ is the unknown complex matrix. The inputs to this problem are $A_{\mathrm{r}}, B_{\mathrm{r}}, A_{\mathrm{i}}, B_{\mathrm{i}}$ and $Y$. The only output for this problem is $X$. The rest of the matrices associated with the complex matrix $C$ are intermediate values and are stored in an IBP depot located near the NetSolve server. To mimic the geographical expanse of the Grid, we placed a NetSolve client application at the University of California, San Diego and experimented with requests to a pool of computational and LoCI servers at the University of Tennessee as illustrated by Fig. 4 .

Fig. 5 compares the performance obtained when the same problem was solved without using the LoCI storage and when using LoCI storage for different matrix sizes. The results show that using LoCI infrastructure in NetSolve helps to improve the performance of data movement for solving problems.

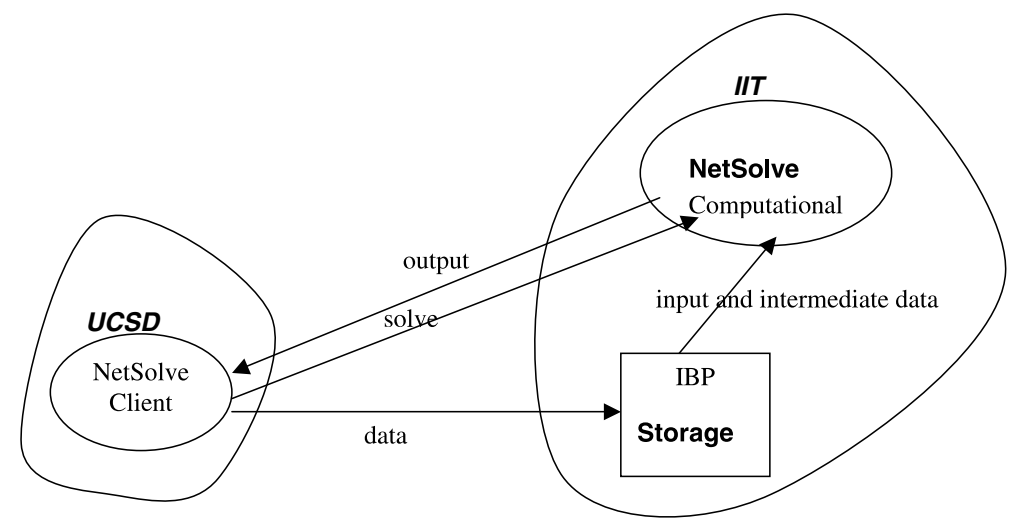

Fig. 4. NetSolve client server storage. 


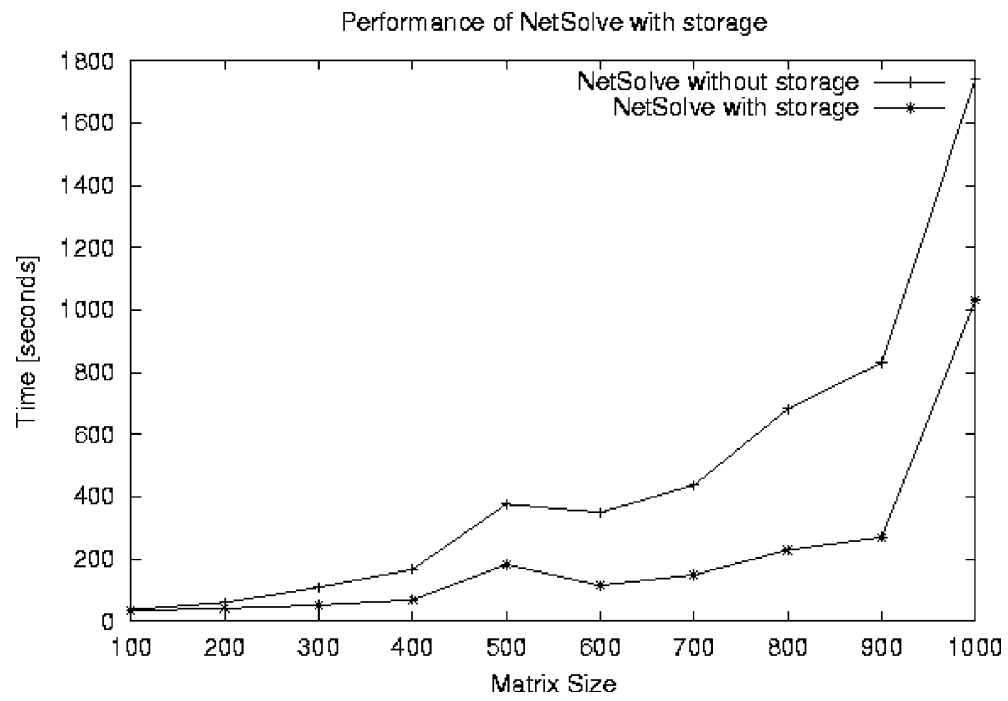

Fig. 5. Performance of NetSolve with and without network storage.
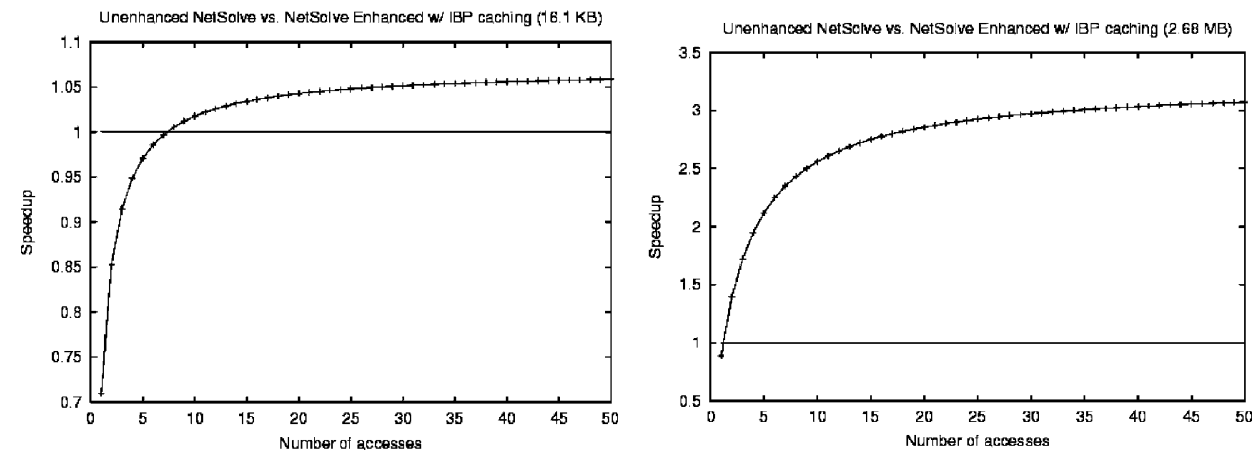

Fig. 6. Results of improved computational efficiency when IBP caching is used with NetSolve.

We also solved another problem involving sparse matrices from the HarwellBoeing collection of the Matrix Market repository to solve system of equations using the MA28 solver library. Fig. 6 illustrates the performance benefits of using LoCI storage in NetSolve improves with the increase in number of accesses to the storage by the NetSolve servers.

\section{Logistical scheduling and the AppLeS project}

The AppLeS project [4,16] focuses on the design and development of Gridenabled high-performance schedulers for distributed applications. The first genera- 
tion of AppLeS schedulers demonstrated that simultaneously taking into account application- and system-level information makes it possible to effectively schedule applications onto computational environments as heterogeneous and dynamic as the Grid. The benefits of that approach have been demonstrated for over a dozen applications (see the AppLeS Web site [17]). However, all those schedulers were tightly embedded within the application itself and thus difficult to re-use. Therefore, the next step was to build on AppLeS principles to provide re-usable software environments that target classes of structurally similar applications. Two such environments, or templates, have been developed so far: the AppLeS Master Worker Application Template (AMWAT) [18] and the AppLeS Parameter Sweep Template (APST) [19]. In the context of the LoCI project, we have investigated and implemented logistical scheduling as part of APST.

APST targets the class of parameter sweep applications (PSAs), i.e., applications structured as large numbers of computational tasks that exhibit little or no synchronization. In addition, we assume that tasks use (and may share) potentially large input datasets, and produce potentially large output datasets. The PSA model is representative of well-known methodologies such as parameter space search techniques, Monte Carlo simulations, and parameter studies. As such, PSAs arise in many fields, including Computational Fluid Dynamics, Bioinformatics, Particle Physics, Discrete-event simulation, Computer Graphics, and many areas of Biology. PSAs are primary candidates for Grid computing given their large scale. Also, due to their loose task synchronization requirements, PSAs are able to tolerate high latencies and faults, both of which are to be expected on the Grid.

Many real-world PSAs are manipulating increasingly large datasets that need to be moved among and staged on distributed Grid storage. Data movement is needed so that distributed compute resources can be utilized, and data staging is needed so that data can be shared and re-used by application tasks. The question is then: How does one make decisions on where to move and where to stage application data in order to minimize application execution time? In this paper we have termed this problem as logistical scheduling, which is key for improving the performance of PSAs on the Grid.

Much research work has been devoted to the problem of scheduling independent tasks. This problem is NP-complete, and several heuristics have been proposed (see [20] for a comparative survey). In this work we have built on three list-scheduling heuristics described in [21]: Min-min, Max-min, and Sufferage. Those heuristics have been shown to be effective when application tasks exhibit affinities with compute resources. This means that some hosts are better for some application tasks, but not for others (e.g., due to specialized hardware, optimized software libraries, etc.). Our key intuition is that the presence of input data on a Grid storage resource "near" a Grid compute resource leads to affinities. In other words, a task has affinity with compute resources which are "near" input data required by that task. This is particularly relevant for PSAs where tasks use and share large datasets over a Grid.

We have made a number of contributions to the PSA scheduling problem on the Grid. First, we have extended the three heuristics in [22] so that they take into 
account logistical issues (input data staging, input data re-use, and data movements). Second, we developed a new heuristic, XSufferage, that exploits some of the structure of the Grid computing platform. Third, we enhanced all four heuristics so that they can be executed in an adaptive fashion: scheduling decisions can be revised periodically at runtime in order to account for dynamic resource conditions. All details on this work can be found in [23]. In that paper we presented many simulation experiments comparing the four list-scheduling heuristics and a greedy self-scheduled work queue algorithm. The main difference between the list-scheduling heuristics and the self-scheduled work queue is that the former require performance predictions (answers to questions like "how long will that task take on that resource?"), whereas the latter does not. One interesting question is then to quantify the impact of performance prediction inaccuracy on the effectiveness of list-scheduling heuristics. We derived two significant conclusions from our simulation results:

(i) XSufferage outperforms other heuristics (and the self-scheduled work queue) by more than $10 \%$ on average when one assumes perfect performance prediction;

(ii) Adaptivity makes it possible for the list-scheduling heuristics to tolerate performance prediction errors (and outperform the self-scheduled work queue).

After developing this adaptive logistical scheduling strategy, we implemented it as part of the APST software. Implementing logistical scheduling requires

(i) fine-grain control of Grid storage resources;

(ii) fine-grain control of Grid compute resources;

(iii) predictions of the expected performance of data transfers and computations on those resources.

Those three requirements are met by IBP, NetSolve, and NWS, which have been described in earlier sections. IBP provides the levels of control required to move and stage application data among distributed IBP storage servers. NetSolve provides a simple way to execute application tasks on remote resources while using data staged in IBP servers as input. Those two mechanisms allow for the implementation of all logistical scheduling decisions made by our adaptive heuristics. By default, if APST is configured to use list-scheduling, it uses the XSufferage heuristic which was proved to achieve the best performance in our simulation experiments. Finally, NWS predictions are the basis for making scheduling decisions. APST uses a combination of NWS predictions as well as historical observations of application performance as input to the list-scheduling heuristics. Finally, the APST software uses several techniques to improve performance, such as multi-threading for latency-hiding and network transfer overlapping. Those techniques, as well as all other implementation details, are described in [19].

In [19] we also described experimental results that corroborate parts of our simulation results. Our main result is that we have shown that list-scheduling is 
indeed practical for real-world Grid computing. Our results, obtained on a Grid testbed containing storage and compute resources in Tennessee, California, and Japan, showed that XSufferage can make use of IBP, NetSolve, and NWS in order to greatly improve application performance over the standard self-scheduled work queue approach. In those experiments, performance prediction errors were of the order of $10 \%$ for compute resources and 30\% for network resources. According to what we observed in our simulation experiments, those errors are well within the bounds of what can be tolerated thanks to the use of adaptivity.

The APST software is an active development project that has grown out of the LoCI activity. We extended it to use other Grid middleware services, including those provided by Globus [25] and Condor [26]. Version 1.1 of the software is freely available from the project's website [24]. We are currently pursuing several new research directions concerning scheduling and performance of PSAs on the Grid platform. For instance, we are investigating scheduling techniques for applications that consist of a partitionable workload (such as applications from bio-informatics). This work is building on scheduling algorithms surveyed in [15]. Also, we are investigating how the ability to change which list-scheduling heuristic is used at runtime can improve the overall performance of PSAs.

\section{Conclusions and future work}

By exposing the intermediate communication state to application or middleware control, Logistical Computing forms a comprehensive approach to Grid computing. Process resources, network resources, and storage resources can be explicitly controlled and scheduled to ensure performance in the face of fluctuating resource availability. In particular, the IBP allows distributed applications to break with the end-to-end communication model achieving better QoS levels through explicit state control. By combining this innovative approach to dynamic storage management with NetSolve and the NWS we have been able to deliver high-performance distributed computing to the end user through the familiar RPC programming model. Our intention is to continue our development of Logistical Computing and to deploy a campus-wide testbed using the Scalable Intracampus Research Grid (SInRG) at the University of Tennessee. Designed to develop a University Grid user community, we are developing a Logistical Computing environment for SInRG both as a means of validating our results, and easing the Grid programming burden.

The software for IBP, NWS, NetSolve, and AppLeS can be found at the following URLs:

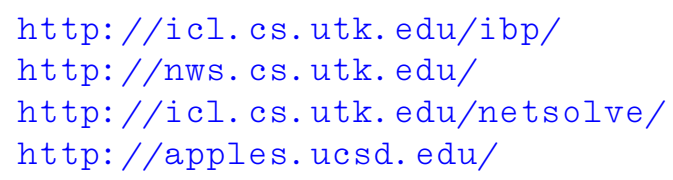




\section{References}

[1] J. Plank et al., The Internet backplane protocol: storage in the network, in: NetStore99: The Network Storage Symposium, Seattle, WA, 1999.

[2] R. Wolski, N. Spring, J. Hayes, The network weather service: a distributed resource performance forecasting service for metacomputing, Future Gener. Comp. Syst. 15 (5-6) (1999) 757-768.

[3] H. Casanova et al., Application-specific tools, in: I. Foster, C. Kesselman (Eds.), The Grid: Blueprint for a New Computing Infrastructure, Morgan Kaufman Publishers, San Francisco, CA, 1998, pp. $159-180$.

[4] F. Berman et al., Application-level scheduling on distributed heterogeneous multiprocessor systems, in: Proceedings of Supercomputing '96, 1996.

[5] H. Casanova, J. Dongarra, Applying NetSolve's network enabled server, IEEE Comput. Sci. Eng. 5 (3) (1998) 57-66.

[6] I. Foster, K. Kesselman, Globus: a metacomputing infrastructure toolkit, Int. J. Supercomp. Applicat. 11 (2) (1997) 115-128.

[7] A. Grimshaw, W. Wulf, et al., The Legion vision of a worldwide virtual computer, Commun. ACM 40 (1) (1997) 39-45.

[8] A. Agbaria, J.S. Plank, Design, implementation, and performance of checkpointing in NetSolve, in: International Conference on Dependable Systems and Networks (FTCS-30 \& DCCA-8), 2000.

[9] M. Swany, R. Wolski, Data Logistics in Networking: The Logistical Session Layer, University of Tennessee, Knoxville, TN, 2001.

[10] A. Geist et al., PVM: Parallel Virtual Machine. A Users' Guide and Tutorial for Networked Parallel Computing, The MIT Press, Cambridge, MA, 1994.

[11] A. Bassi et al., Internet Backplane Protocol: API 1.0, University of Tennessee, Computer Science Department, 2001.

[12] W. Elwasif et al., IBP-mail: controlled delivery of large mail files, in: NetStore '99: Network Storage Symposium, 1999, Internet2, http://dsi.internet2.edu/netstore99.

[13] R. Wolski, Forecasting network performance to support dynamic scheduling using the Network Weather Service, in: Proceedings of the 6th IEEE Symposium on High Performance Distributed Computing, IEEE Computer Society Press, Los Alamitos, CA, 1997, pp. 316-325.

[14] I. Foster, C. Kesselman (Eds.), The Grid: Blueprint for a New Computing Infrastructure, Morgan Kaufman Publishers, San Francisco, CA, 1998, p. 677.

[15] T. Hagerup, Allocating independent tasks to parallel processors: an experimental study, J. Parallel Distrib. Comput. 47 (1997) 185-197.

[16] F. Berman, R. Wolski, The AppLeS Project: a status report, Proceedings of the 8th NEC Research Symposium, Berlin, Germany, May, 1997.

[17] http://apples.ucsd.edu.

[18] G. Shao, Adaptive Scheduling of Master/Worker Applications on Distributed Computational Resources, PhD thesis, University of California, San Diego, May, 2001.

[19] H. Casanova, G. Obertelli, F. Berman, R. Wolski, The AppLeS parameter sweep template: user-level middleware for the Grid, in: Proceedings of SuperComputing 2000 SC'00, November 2000.

[20] T.D. Braun, H.J. Siegel, N. Beck, L. Boloni, M. Maheswaran, A.I. Reuther, J.P. Robertson, M.D. Theys, B. Yao, D. Hensgen, R.F. Freund, A comparison study of static mapping heuristics for a class of meta-tasks on heterogeneous computing systems, in: Proceedings of the 8th Heterogeneous Computing Workshop (HCW'99), April 1999, p. 15-29.

[21] O.H. Ibarra, C.E. Kim, Heuristic algorithms for scheduling independent tasks on nonindentical processors, J. ACM 24 (2) (1977) 280-289.

[22] M. Maheswaran, S. Ali, H.J. Siegel, D. Hensgen, R. Freund, Dynamic matching and scheduling of a class of independent tasks onto heterogeneous computing systems, in: 8th Heterogeneous Computing Workshop (HCW'99), April 1999, p. 30-44.

[23] H. Casanova, A. Legrand, D. Zagorodnov, F. Berman, Heuristics for scheduling parameter sweep applications in Grid environments, in: Proceedings of the 9th Heterogeneous Computing Workshop (HCW'00), May 2000, p. 349-363. 
[24] The APST homepage, http://grail.sdsc.edu/projects/apst/.

[25] Globus homepage, http://www.globus.org.

[26] J. Basney, M. Livny, Deploying a high throughput computing cluster, in: High Performance Cluster Computing, vol. 1, Prentice Hall, May, 1999, Chapter 5. 